\title{
CLASSICAL CONDITIONING MEDIATED BY THE RED NUCLEUS IN THE CAT ${ }^{1}$
}

\author{
NAKAAKIRA TSUKAHARA, ${ }^{*} \ddagger$ YOICHI ODA, ${ }^{*}$ AND TATSUTO NOTSU* \\ * Department of Biophysical Engineering, Faculty of Engineering Science, Osaka University, Toyonaka, Osaka and $\ddagger$ National Institute for \\ Physiological Sciences, Myodaiji, Okazaki, Japan
}

\begin{abstract}
We have attempted to develop a behavioral and neuronal model for classical conditioning in the corticorubrospinal system. A conditioned stimulus (CS) was applied to the cerebral peduncle (CP) in cats which had lesions that interrupted the corticofugal fibers caudal to the red nucleus. The unconditioned stimulus (US) was an electric shock to the skin of the forelimb that produced flexion of the limb. After pairing of the CS and US in close temporal association, an initially ineffective stimulus to the cerebral peduncle was found to give rise to the flexion of the elbow. Extinction of the conditioned response was achieved by applying the CS alone or by reversing the sequence of the stimuli (US-CS: backward pairing). Furthermore, the US alone did not produce an increase in the effectiveness of the CS stimulus. Finally, pairing the fixed CS stimuli with the US at random intervals did not produce any increase in performance in response to the CS. In these respects, the observed behavioral modification has the features of associative conditioning.

Because the thresholds for and the strength of elbow flexion induced by stimulation of the nucleus interpositus of the cerebellum were identical in the experimental and control animals, the interpositorubrospinal system cannot be the site of the plastic change. Since the conditioned response is most probably mediated by the corticorubrospinal system, it is likely that a modification of the corticorubral synapses underlies this behavioral change.
\end{abstract}

There is a large body of work dealing with the neuronal correlates of classical conditioning (Kandel and Spencer, 1968; Woody and Brozek, 1969; Thompson et al., 1972; Kandel, 1976; Thompson et al., 1978). In order to understand the neuronal basis of learning, it is necessary, first of all, to characterize the mode of behavioral modification that occurs, for example, in classical conditioning. Then, it is necessary to identify the neuronal circuit responsible for the behavior and to localize the primary sites in which the changes that underlie the behavioral modification occur. Finally, the cellular mechanisms of the behavioral modification should be analyzed.

In the mammalian central nervous system, it is generally difficult to identify the neuronal circuitry involved in any form of complex learning behavior. Therefore, it is important to select a simplified preparation in which the identification of the relevant neuronal circuit and the isolation of the primary site of the behavior are more accessible.

The red nucleus (RN) offers certain advantages for behavioral studies, since much of its synaptic organization and network connections have been well characterized (Allen and Tsukahara, 1974). Furthermore, recent

\footnotetext{
${ }^{1}$ This work was supported by a grant from the Ministry of Education, Science and Culture of Japan.
}

studies have shown that corticorubral synapses are capable of a remarkable degree of plasticity. In addition to lesion-induced sprouting which occurs after lesions of the nucleus interpositus (IP) of the cerebellum (Tsukahara et al., 1974, 1975; Murakami et al., 1977a, b; Nakamura et al., 1974; Hanaway and Smith, 1978), sprouting and the formation of new functional synapses can occur after cross-innervation of the forelimb flexor and extensor nerves (Tsukahara and Fujito, 1976; Tsukahara, 1978), which suggests that sprouting may occur under more physiological conditions than those following brain lesions.

In this paper, we have attempted to use the phenomenon of synaptic plasticity of the corticorubral synapses as an element in the reconstruction of a simplified behavioral paradigm. There is already evidence that midbrain structures are important for classical conditioned avoidance responses (Doty, 1969; Thompson et al., 1972) and Smith (1970) has reported that rubral lesions abolish a conditioned forelimb flexion response when a tone is used as the conditioning stimulus (CS) and an electric shock to the forelimb serves as the unconditioned stimulus (US). We have attempted, therefore, to simplify this paradigm by limiting both the afferent pathway involved and the conditioned behavior mediated by the red nucleus by applying the CS to the corticorubral fibers as 
they pass through the cerebral peduncle (CP). The CS is delivered in the form of electric pulses in a preparation in which the cerebral corticofugal outflow is largely restricted to the corticorubrospinal pathway by section of the cerebral peduncle caudal to the red nucleus. In this preparation, we have found that after paired conditioning (by stimulating the CP as the CS and applying electric shocks to the skin of the forelimb as the US in close temporal association) initially ineffective stimuli to the corticorubral fibers gives rise to forelimb flexion; this has never been observed after random control, CS-US stimulation, after CS alone, after US alone, or after backward pairing of the two stimuli. Furthermore, by testing the flexion responses induced by another monosynaptic excitatory input to the red nucleus (from the nucleus interpositus (IP) of the cerebellum), evidence has been obtained which suggests that the primary sites of the conditional change are located at the corticorubral synapses.

A preliminary report of some of these results has been published elsewhere (Tsukahara et al., 1979).

\section{Materials and Methods}

Surgical procedures. Adult cats were used for all of the experiments. Under sodium pentobarbitone anesthesia, the animals were mounted on a stereotaxic frame and the corticofugal fibers on the left side were destroyed electrolytically by passing current at 13 separate points at each of three rostrocaudal levels corresponding to A 0 , A 0.5, and A 1.5. From later histological examination of the brains, it was verified that the lesions were in fact in the cerebral peduncle and in the pontine nuclei of the left side as shown diagrammatically in Figure $1 B$.
Bipolar electrodes, made of a pair of acupuncture needles insulated except at their tips, were inserted into the cerebral peduncle of the left side (at A. 8.5) and cemented to the skull. In some cases, a similar electrode was also implanted into the right nucleus interpositus (IP). A pair of silver electrodes was used to provide the unconditioned stimulus to the shaved skin of the right forearm (Fig. 1A). After these surgical procedures, the animals were kept in their cages for several days.

Training procedures. Seven days after surgery, the cats were brought into the training room. They were restrained in bags with holes from which their heads and right forelimbs protruded. They were then mounted in a specially designed frame with the right shoulder affixed to the frame to maintain the forelimb at the predetermined resting position. In this situation, the cats could readily flex their elbows from the resting position (Fig. $1 C)$.

Formal training started 4 days after an initial pretraining period which enabled the animals to become accustomed to the experimental room and allowed us to determine the appropriate intensity of the CS. Current intensity for the CS was selected in the following way. First, the stimulus intensity required to evoke elbow flexion was determined; next, the proportion of positive responses (out of a total of 20 trials) was estimated; then, this was defined as the "score of performance" for that current intensity. A positive response was defined as a flexion at the elbow joint of more than $0.05^{\circ}$, which was the maximum resolution of our recording system for elbow movements. The "score of performance" increased as the CS stimulus intensity was increased as shown in
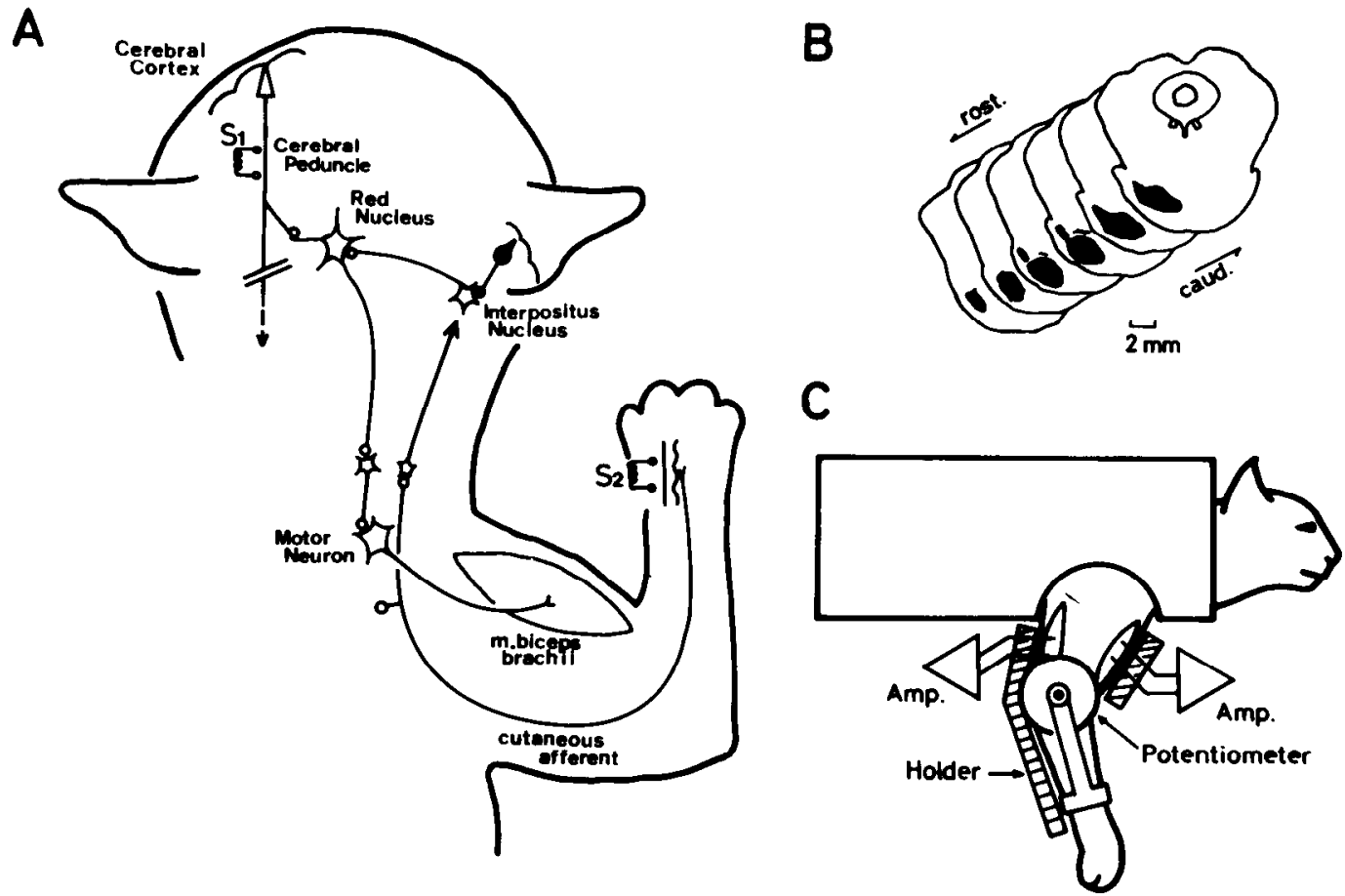

Figure 1. A diagram to illustrate the experimental arrangement. A, A diagram of the principal neuronal circuits involving the red nucleus. $S 1$, conditioned stimulus; $S 2$, unconditioned stimulus. $B$, Drawings of histological sections through the midbrain to illustrate the lesions in the cerebral peduncle. $C$, The setup used for measuring movements at the elbow joint. Joint angle was measured by a potentiometer. Electrical activities from the biceps and triceps brachii muscles were amplified by amplifiers (Amp.). Holder, a device to hold the cat's forearm in the predetermined position. 
Figure 2E. A current intensity that produced a $50 \%$ "score of performance" was accepted as " $50 \%$ current" and the average for this $50 \%$ current (during four consecutive pretraining days) was determined. Seventy-five percent of this $50 \%$ current intensity was used during the subsequent training sessions for the CS.

During conditioning, the animals were given 120 periods of training per day, with CS-US paired trials; once every five trials, the CS was presented alone. The intertrial interval was fixed at $30 \mathrm{sec}$. The CS was a train of five pulses each with a duration of $0.1 \mathrm{msec}$ and was presented at intervals of 2 msec (Fig. $2, A, C$, and $D$ ). In order to avoid electrode polarization, the polarity of the pulse was automatically reversed after every train of pulses. The US was an electric pulse with a duration of $10 \mathrm{msec}$; this was preceded by the CS some 60 to 200 msec earlier (but most often $100 \mathrm{msec}$ ) (Fig. 2B). CS-US pairing of 120 trials, at intervals of $30 \mathrm{sec}$, constituted the training session on any given day. The ratio of positive responses in 24 such trials, without the US, provided the score of performance for that day.

Control procedures consisted of: (1) presentation of the CS alone; (2) the US alone; (3) backward pairing of the stimuli; (4) random presentation of the two stimuli. In the case of the random stimulus presentations, the animals received the CS and US in the same session, in random sequence; the CS was presented at regular intervals of $30 \mathrm{sec}$, but the US was presented randomly anywhere from 0 to $30 \mathrm{sec}$ after the onset of the CS. This random US stimulus presentation was achieved by controlling the stimulator by a microcomputer in which random numbers had been stored in memory.

Data acquisition and analysis. A potentiometer was attached to the elbow joint (Fig. $1 C$ ). The output of this potentiometer was connected to an amplifier and the amplified signals were recorded by a pen recorder. The resolution of the movement at the elbow joint was $0.05^{\circ}$. Teflon-coated needles were inserted into the biceps and triceps brachii muscles of the right arm for electromyographic (EMG) recording. The electromyograms were amplified and displayed on the pen recorder simultaneously with the associated elbow movements. The electromyogram was also displayed on an oscilloscope and photographed. The speed of the pen recorder was automatically controlled by a microcomputer throughout the training session.

Histological procedures. At the end of each experiment, the animals were anesthetized with sodium pentobarbitone and small electrolytic lesions were made by passing current through the stimulating electrodes. Then
A
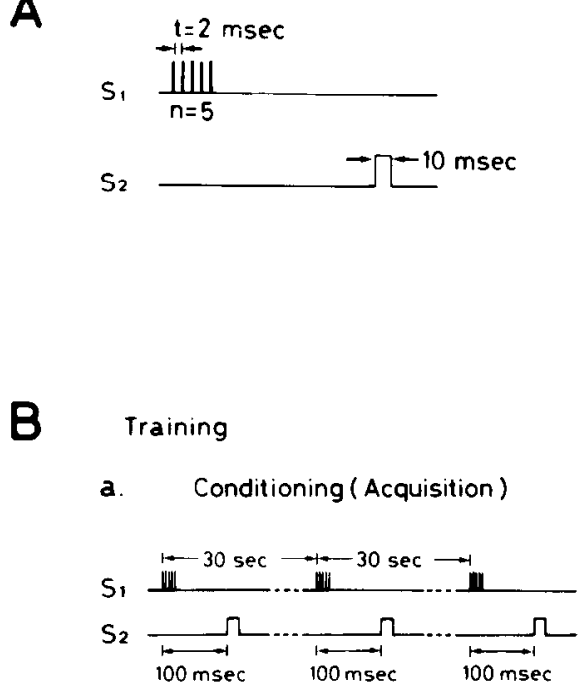

b. Random

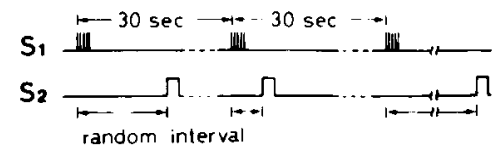

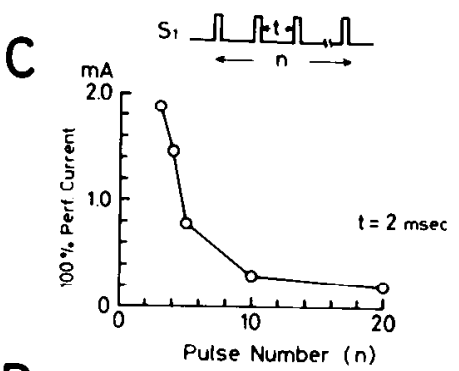

D

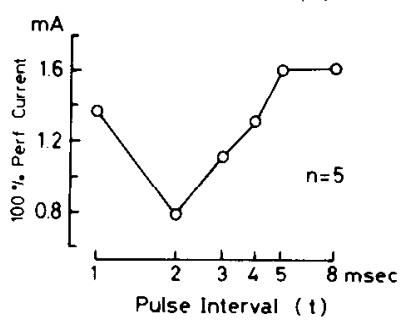

E

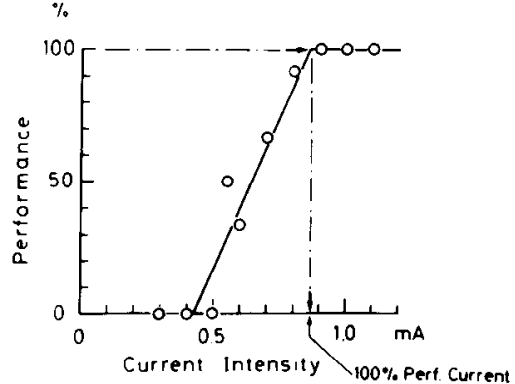

Figure 2. Parameters of stimulation. A, Stimulus parameters for conditioning. $S 1$, conditioned stimulus; $S 2$, unconditioned stimulus. $B$, Two different modes of CS-US pairing: $a$, conditioning (acquisition) mode in which the $S 1$ is applied at regular intervals of $30 \mathrm{sec}$ followed by $S 2$ with a standard delay of $100 \mathrm{msec} ; b$, random control in which the CS is regularly applied, whereas the US is spaced randomly. $C$, Ordinate, $100 \%$ performance current; abscissa, number of pulses in the train of CS stimuli. $D$, Ordinate, $100 \%$ performance current; abscissa, time interval of successive pulses of CS. $E$, The relation between the intensity of the CS and the score of performance. 
they were perfused with $10 \%$ formalin, and the brains were removed and prepared for histological examination to verify the position of stimulating electrodes and the completeness of the lesion of the cerebral peduncle.

\section{Results}

Figure 3, $A$ to $C$, shows an example of elbow flexion on the 1st (Fig. 3, $A$ and $B$ ) and 7th days (Fig. $3 C$ ) after CS-
A

B
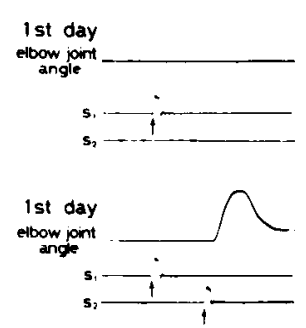

C

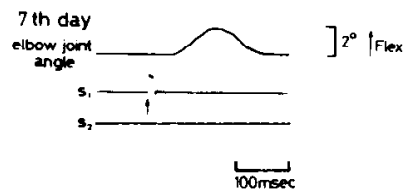

D
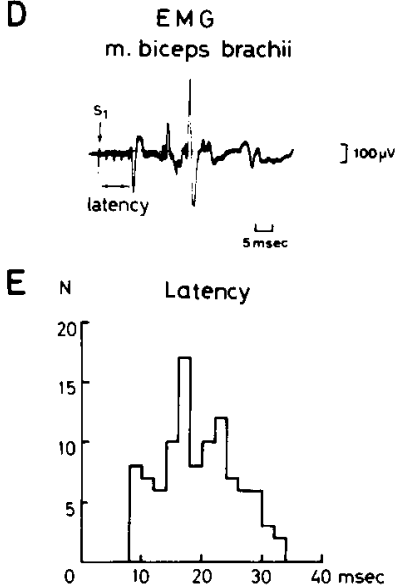

Figure 3. Sample records of conditioning. $A$ and $B$, Flexion of the elbow joint from the 1st day of training. S1, onset of CS; $S 2$, onset of US. In $A$, the CS produced no response. In $B$, the CS-US produced flexion in response to US. $C$, Flexion of elbow joint in the same animal but after 7 days of training. Now the CS alone produces elbow flexion. Arrows indicate the onset of the stimuli. The time calibration and the flexion of $2^{\circ}$ in $C$ also apply to $A$ and $B$. D, Electromyogram (EMG) of biceps brachii muscles. $S 1$, onset of the first pulse in a train of five pulses. $E$, Frequency distribution of the latency of the first spike in the electromyographic record as measured as in $D$ (from two scparate cats). Ordinate, number of spikes to CS stimuli; abscissa, latencies in milliseconds.
US paired conditioning. On the 1st day of training, the CS alone produced no response, whereas the US by itself produced an elbow flexion. After CS-US pairing for 7 days, the CS alone produced a flexion at the same stimulus intensity as was used before (i.e., as in Fig. $3 A$ ). An example of the electrical activity recorded from the biceps muscle during one of the training sessions is illustrated in Figure $3 D$; in this figure, $S 1$ is the onset of the first in a train of five pulses at intervals of $2 \mathrm{msec}$. The latencies of the first spike observed in the electromyographic records from the onset of the first pulse of the CS were measured and the frequency distribution of these latencies is illustrated in Figure $3 E$ for two representative records made on the 9th day in one cat and on the 13th day of training in another animal. The latencies ranged from 8 to 34 msec. Before training, the CS (at the higher intensities used for training) produced spikes in the biceps muscles with significantly longer latencies but within the range of 12 to $32 \mathrm{msec}$.

Figure $4 A$ is an example of the time course in the change of "score of performance" after training. The score of performance, as defined by the probability of positive responses with a fixed CS stimulus intensity, increased gradually until it reached a plateau after about 7 days. With extinction (produced by reversing the sequence of the stimuli, i.e., US-CS), the score of performance returned to the pretraining level. In parallel with the increase in the score of performance, the minimum current intensity required to produce $100 \%$ of the score of performance ( $100 \%$ current or $100 \%$ performance current) progressively decreased (Fig. $4 B$ ). With extinction, it returned to the pretraining level. The $100 \%$ current intensity was measured at the end of each day's training session by changing the stimulus intensities and by measuring the score of performance in 60 trials. Figure $4 C$ shows an example of the relationship between the score of performance and the respective CS current, in the
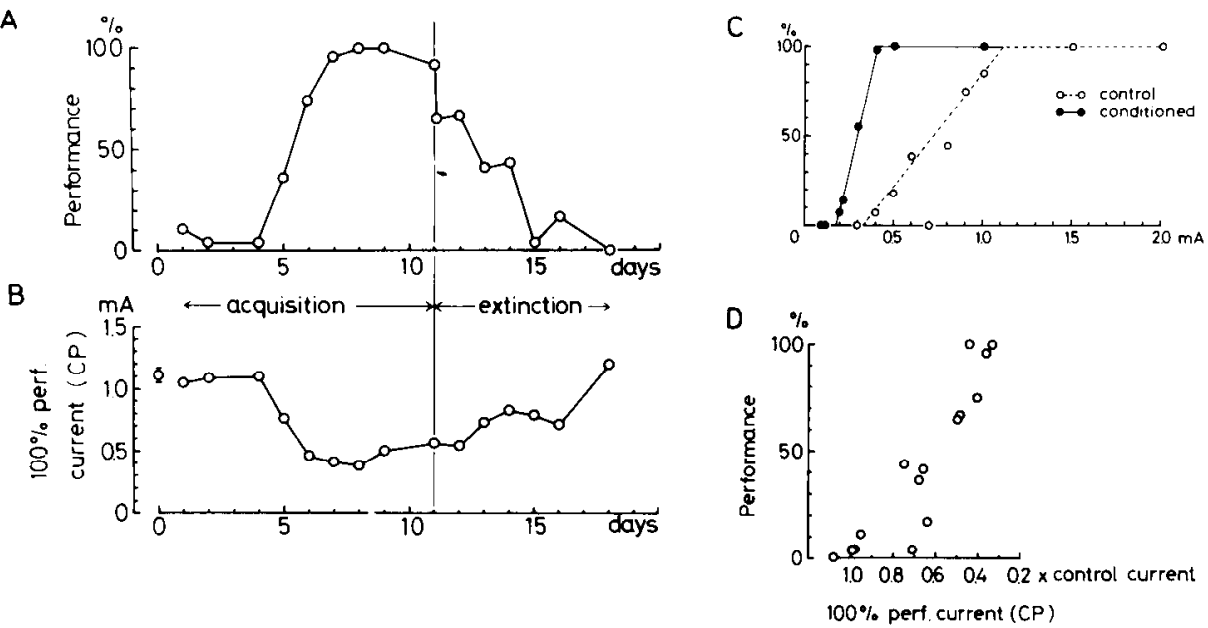

Figure 4. The time course of associative learning. A, The score of performance during forward and backward conditioning. Ordinate, score of performance; abscissa, day after onset of training. CS-US interval, 100 msec. After the 11th day of CS-US pairing, the stimulus sequence was reversed (i.e., US-CS) with an interval of 900 msec. $B$, Minimum current for eliciting $100 \%$ performance ( $100 \%$ current) plotted against time as in $A$. C, Score of performance (mean of 60 trials) with changes of CS current intensities. $O$, before training. $O$, after the establishment of conditioning (mean of $7 \mathrm{th}$, 8th, and 9 th days after onset of training). $D$, The relationship between the score of the performance and the $100 \%$ current shown in $A$ and $B$. Ordinate, score of performance; abscissa, $100 \%$ current normalized against the control current. 
same animal, before and after training. It is evident that the slope of the curve became steeper after training, as compared to pretraining (see "Discussion"). From this curve, a figure for the " $100 \%$ current" was determined. When the estimate of $100 \%$ current at the end of the session is compared to the score of performance during the whole conditioning period, it is obvious that there is a good correlation (with a correlation coefficient, $r=$ -0.907) between these two parameters as shown in Figure $4 D$. Therefore, either of these parameters could be used as a measure of the acquisition and extinction of the conditioned response.

Furthermore, in parallel with the increase in the score of performance, the degree of the observed elbow flexion also increased as shown in Figure $5 A$. By varying the stimulus intervals in the CS-US pairing, the score of performance was measured and plotted as in Figure $5 B$. Here one can see that the most effective stimulus interval was about 100 msec.

The results from 10 animals are summarized in Figure $6, A$ and $B$. The mean score of performance for the 10 cats reached its peak on the 9th day after the onset of training. Similarly, the $100 \%$ current decreased to a minimum on the same day (Fig. $6 \mathrm{~B}$ ). The score of performance or $100 \%$ current on the 1st day and that of the 9th day are significantly different $(p<0.001)$.

The extinction experiments were performed in three ways; these are illustrated in Figure $6 C$. First, by presenting the CS alone (open circles in Fig. $6 \mathrm{C}$ ); second, by backward pairing with an interval of $900 \mathrm{msec}$ (open triangles in Fig. 6C); and third, backward pairing at intervals of $10 \mathrm{sec}$ (open squares in Fig. $6 C$ ). The time course of the extinction in all three procedures is similar both for the score of performance (Fig. $6 C$ ) and for the $100 \%$ current (Fig. $6 D$ ).

Random control experiments were also performed in two cats, and the results from these two cases are illustrated in Figure 7, $A$ and $B$. The CS presented at regular intervals of $30 \mathrm{sec}$ was followed by the US presented randomly (as determined by the microcomputer-controlled stimulating system). With this mode of stimulation, neither the score of performance nor the $100 \%$ current changed appreciably with training. In a third cat, the US was presented by itself for 10 days. In this case, the $100 \%$ current did not show any appreciable change (see Fig. $7 \mathrm{C}$ ); therefore, it seems likely that the behavioral modification is to be considered an example of classical conditioning.
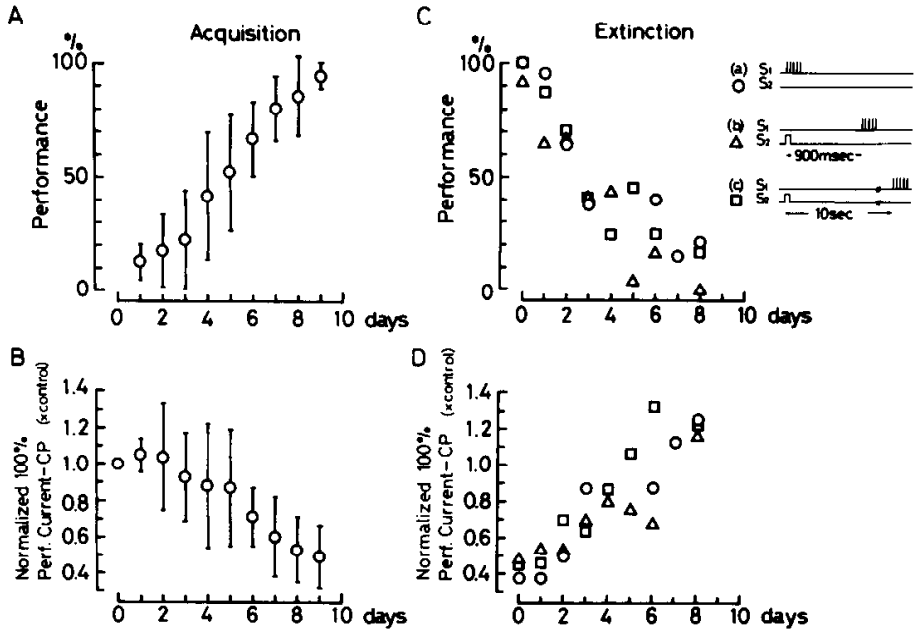

Figure 6. Mean time course of acquisition and extinction of the conditioned responses. $A$ and $B$, Summarized data from 10 cats. $A$, Ordinate, mean and SD of the score of performance as illustrated in Figure $4 A$. Abscissa, day after onset of training. $B$, As in $\mathrm{A}$, but the normalized current intensity for producing $100 \%$ score of performance is plotted against the day after the onset of training. $C$ and $D$, Time course of extinction. $O$, CS alone; $\triangle$, US-CS sequence with an interval of $900 \mathrm{msec}$; $\square$, USCS sequence with an interval of $10 \mathrm{sec} . C$, Score of performance during extinction. $D$, Current intensity producing $100 \%$ performance as a ratio of the control current, plotted against the days during extinction.
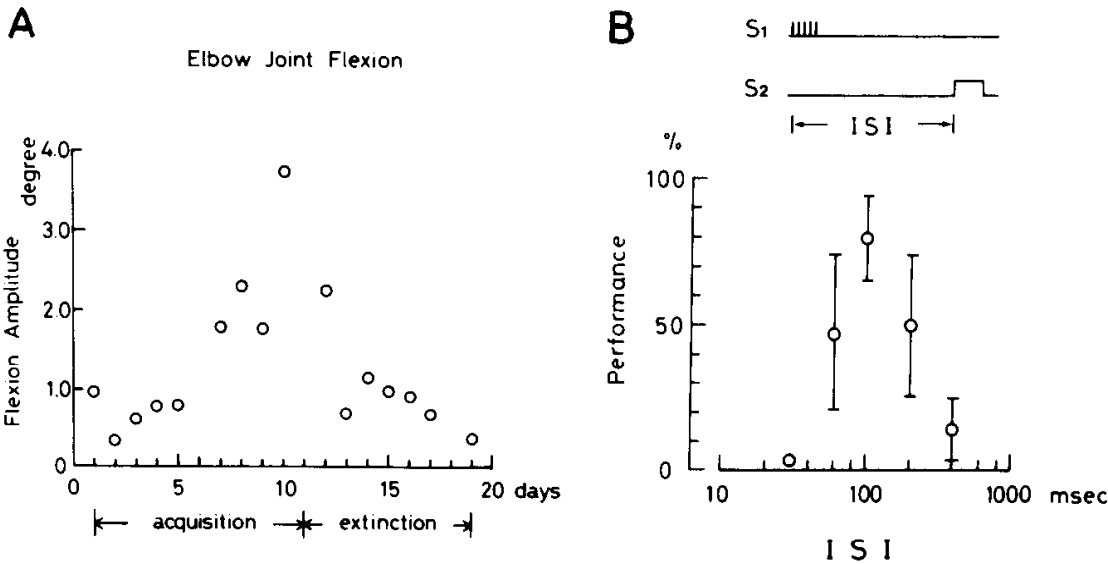

Figure 5. Increase of degree of flexion during conditioning and optimal CS-US interval. $A$, Ordinate, flexion of the elbow joint in degrees; abscissa, days after onset of training (acquisition) and extinction by US-CS with an interval of 10 sec. $B$, Ordinate, score of performance; average and SD of mean score of performance on the 7th day after the onset of conditioning (derived from a total of 21 cats). The numbers of cats used for each point were as follows: one for the 30-msec, five for the 60-msec, nine for the 100 -msec, four for the $200-\mathrm{msec}$, and two for the 400 -msec interval. Abscissa, interstimulus intervals (ISI) of CS-US as shown diagrammatically in the inset. 
A

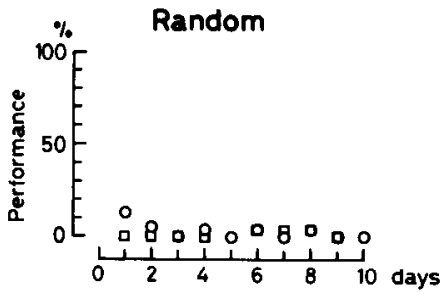

B

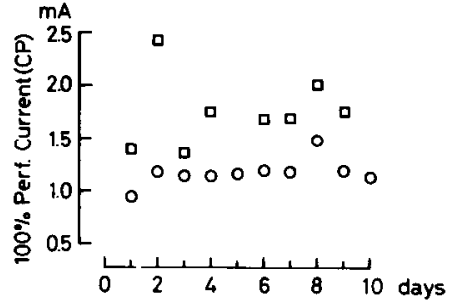

C

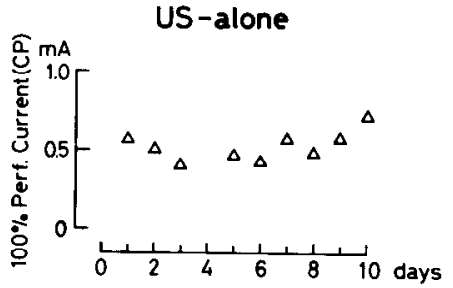

Figure 7. Random control and control with US alone. $A$ and $B$, Random control. $A$, Ordinate, score of performance during random control experiments in two cats with the stimulus parameters shown in Figure $2 B b$. Abscissa, day after CS-US pairing with random intervals. $B$, Ordinate, $100 \%$ performance current in the same cats as in $A$, using the same symbols for the two cats; abscissa, days after CS-US pairing with random intervals. $C$, US alone. Ordinate, $100 \%$ performance current; abscissa, day after onset of conditioning with US alone.

We have examined the possible sites at which the underlying neuronal changes occur during the establishment of the conditioned response in the following way. As will be discussed more fully under "Discussion," the most likely pathway involved in the conditioned response is the corticorubrospinal system. If this is the case, then the observed changes must take place somewhere within this pathway. And, since the red nucleus receives another monosynaptic excitatory input-from the nucleus interpositus (IP) of the cerebellum by way of the interpositorubral pathway (Toyama et al., 1970 and Fig. $8 A$ ), we may ask whether the efficacy of transmission along the interpositorubrospinal pathway is increased as the efficacy of transmission in the corticorubrospinal pathway is enhanced by the CS-US pairing.

Figure $8 B$ illustrates the time course of the acquisition of the conditioned responses evoked by stimulating the cerebral peduncle as the CS. There is a significant decrease $(p<0.02)$ of $100 \%$ current. In the same group of cats, stimulating electrodes were implanted in the IP nucleus of the contralateral side, and the $100 \%$ performance current was tested with the same trains of pulses used for the $\mathrm{CP}$ stimulus during the development of the conditioned response with CP stimulation. From the figure (Fig. $8 C$ ), it is evident that there is no significant decrease $(p>0.40)$ in the $100 \%$ current required for IP stimulation and, indeed, in some cats, a slight increase in the $100 \%$ current was observed. This result indicates that the efficacy of transmission along the interpositorubrospinal pathway was not improved despite the marked enhancement in transmission along the corticorubrospinal pathway. On this basis, it seems reasonable to suggest that the primary site of the change underlying the $\mathrm{CP}$-conditioned response is not located caudal to the red nucleus. Furthermore, it is unlikely that the CS leads to a general increase in excitability (e.g., a conductance decrease or a decrease in the threshold of the cells in the red nucleus) (see below). Thus, the most probable locus of the induced neuronal change appears to be the corticorubral synapses.

\section{Discussion}

The present study has shown that training with a CS to the cerebral peduncle paired with an US to the skin of the forearm results in an enhancement in the efficacy of transmission along the corticofugal pathway to the forearm flexor motoneurons in response to the CS. Extinction experiments exclude the possibility that the enhanced efficacy of transmission is simply due to some change in the mode of presentation of the CS (e.g., in the properties of the electrodes in the CP). Similarly, we may exclude such phenomena as lesion-induced sprouting of corticorubral fibers following surgical lesions of the CP. Control experiments involving random presentation of the CSUS stimuli, together with several other controls, have served to establish that the behavioral modification falls squarely within the broad category of associative learning.
A

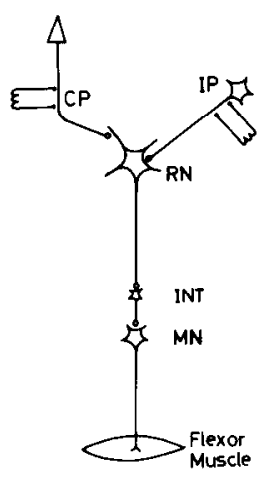

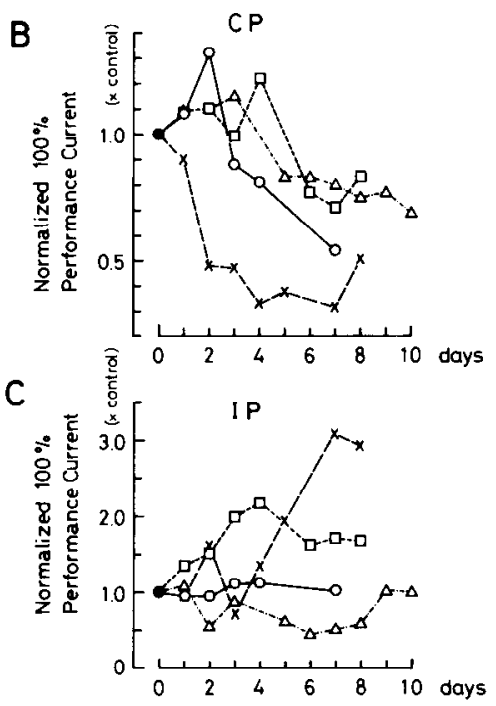

Figure 8. Primary site of conditioned changes. $A, C P$, cerebral peduncle, $I P$, interpositus nucleus of the cerebellum, $R N$, red nucleus, $I N T$, interneurons interpolated in the rubrospinal system, $M N$, flexor motoneurons. $B$, Current intensity of $C P$ stimulation for producing $100 \%$ performance to CS. $C$, Current intensity producing $100 \%$ performance by stimulation of $I P$ with the same train of pulses and in the same cats as used in $B$. The data from four cats are illustrated by different symbols common to $B$ and $C$. Ordinates for $B$ and $C$, normalized $100 \%$ performance current; abscissa, day after onset of conditioning of CS to $C P$ and US to the skin of the forearm. 
The pathway mediating the conditioned response in this experimental paradigm is considered to be relatively simple in view of the fact that the corticofugal fibers were surgically eliminated just caudal to the red nucleus and also because the CS electrodes were implanted within the cerebral peduncle which contains corticorubral fibers that are presynaptic to the cells in the red nucleus. Our CP lesions were accurate enough to exclude the possible contribution of the corticospinal tract. On these grounds, we are confident that the main pathway mediating the conditioned response is the corticorubrospinal system. The latency of electrical activity elicited in the biceps muscle in response to the CS also supports this view; the shortest latency recorded in the electromyograms from the biceps brachii muscle was $8 \mathrm{msec}$, which accords well with the shortest time required for the transmission of impulses along the corticorubrospinal pathway to the muscle. This may be calculated in the following way: (1) $1 \mathrm{msec}$, for conduction from the CP to the red nucleus and the onset of corticorubral EPSPs (Tsukahara and Kosaka, 1968); (2) $2 \mathrm{msec}$, from the onset of the corticorubral EPSPs to the initiation of impulses in the neurons of the red nucleus, assuming that the neurons are excited during the rising phase of the corticorubral EPSPs which have a mean time-to-peak of $3.6 \mathrm{msec}$ (Tsukahara et al., 1975); (3) $2 \mathrm{msec}$, from the onset of spike initiation in the neurons of the red nucleus to the onset of EPSPs in the forelimb motoneurons (Illert et al., 1976); (4) $3 \mathrm{msec}$, from the onset of EPSPs in the motoneurons to the initiation of electrical activity in the biceps muscle. This total of $8 \mathrm{msec}$ is considered to be the shortest possible time.

The neuronal changes that occur after conditioning do not appear to involve any detectable alteration in neuronal elements caudal to the red nucleus, since the efficacy of transmission along the interpositorubrospinal pathway does not change, whereas there is a remarkable increase in transmission efficacy in the corticorubrospinal system. This suggests that the substrates of the conditioned change are to be found in either the terminals of the corticorubral fibers, in transmission between these fibers and the neurons in the red nucleus, or in the dendritic spines upon which the corticorubral fibers synapse. A change in postsynaptic excitability or in the threshold of the postsynaptic neurons seems unlikely. After the establishment of conditioning, the relationship between the "score of performance" and the intensity of the CS stimulus changed in the manner indicated in Figure $4 C$. The change in the shape of this curve is accounted for satisfactorily by assuming a change in corticorubral synaptic transmission rather than by assuming an increase in postsynaptic excitability by the following model.

First, it is assumed that the input-output curve as shown in Figure $4 C$ is determined by the behavior of essentially the entire population of relevant neurons in the red nucleus. For the sake of simplicity, it is also assumed that the current intensity of the CS is related linearly to the number of corticorubral fibers excited and that, similarly, the "score of performance" of forelimb flexion is related linearly to the number of red nucleus neurons innervating the forelimb that are excited. Let $N$ represent the number of such neurons and $C$ the number of relevant corticorubral synapses; then of $N$ red nucleus neurons, $n$ will be excited by $m$ fibers, where $m$ is the proportion of corticorubral fibers activated within the total population of fibers $(M)$. If we assume a randomly connected network in which $M$ corticorubral fibers make synaptic contacts at random with $N$ red nucleus neurons, then the probability that a certain fraction $(x)$ of the input of corticorubral fibers to a given red nucleus neuron will be excited (out of a total of $C$ synapses when $m$ corticorubral fibers are stimulated out of a total of $M$ ) will be given by the following equation (Griffith, 1971).

$$
P(x, m)=\left(\begin{array}{l}
C \\
x
\end{array}\right)\left(\frac{m}{M}\right)^{x}\left(1-\frac{m}{M}\right)^{c-x}
$$

Furthermore, the fraction of excited red nucleus neurons, $n$, out of the total red nucleus neurons, $N$, can be expressed by the following equation:

$$
\frac{n}{N}=\sum_{x=\theta}^{c} \frac{C !}{x !(C-x) !}\left(\frac{m}{M}\right)^{x}\left(1-\frac{m}{M}\right)^{C-x}
$$

where $\theta$ denotes the threshold of the red nucleus neurons (and assuming, of course, that all red nucleus neurons have the same threshold value).

Figure 9 illustrates a family of curves in which $\theta$ is kept constant while $C$ changes (Fig. 9A) or $C$ is kept constant while $\theta$ changes (Fig. $9 B$ ); these two situations correspond to the experimental conditions in which some change occurs at the corticorubral synapses in the former and in which there are excitability changes in red nucleus neurons in the latter. It is evident in Figure $9 B$, that when $C$ is kept constant, the slope of the sigmoid curve for the input-output relation does not change appreciably with changes in threshold. On the other hand, the slope of the sigmoid curve changes quite dramatically if $C$ varies and the threshold is kept constant. The latter accords with the observed experimental findings, and for this reason, it seems reasonable to conclude that there is no appreciable change in the threshold or excitability of
A

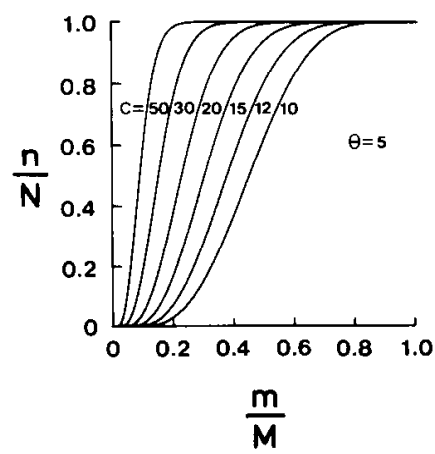

B

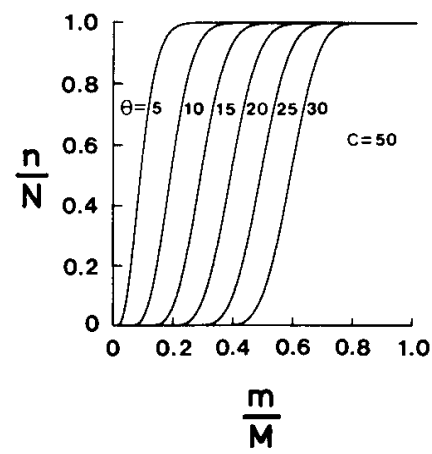

Figure 9. Input-output relationships in the hypothetic neuron pool of the red nucleus. Ordinates, number of excited red nucleus neurons, $n$, as a ratio of the total number $(N)$ of red nucleus neurons. Abscissa, number of excited corticorubral fibers, $m$, as a fraction of the total $(M)$ corticorubral fibers. $A$, Threshold, $\theta$, remains constant, whereas $C$ changes. $B, C$ remains constant, whereas $\theta$ changes; see text for a description of this model. 
the red nucleus neurons after conditioning and that the conditioned response is attributable to changes in either the structural or functional properties of the pre- and/or postsynaptic elements at the corticorubral synapses.

The stability of the conditioned state, which may last for several weeks, suggests that the neuronal substrates of conditioning are to be sought in some persistent structural or functional change in the corticorubral synapses. In this context, it is relevant to recall that sprouting and the formation of functional corticorubral synapses can take place even in the absence of brain damage (Tsukahara and Fujito, 1976; Tsukahara, 1978, 1981), and when viewed in this way, the present experimental paradigm provides an almost unique opportunity for studying the neuronal basis of conditioning in the mammalian central nervous system.

\section{References}

Allen, G. I., and N. Tsukahara (1974) Cerebrocerebellar communication systems. Physiol. Rev. 54: 957-1006.

Doty, R. W. (1969) Electrical stimulation of the brain in behavioral context. Annu. Rev. Psychol. 20: 289-320.

Griffith, J. S. (1971) Mathematical Neurobiology. Academic Press, New York.

Hanaway, J., and J. Smith (1978) Sprouting of corticorubral terminals in the cerebellar deafferented cat red nucleus. Soc. Neurosci. Abstr. 4: 1507.

Illert, M., A. Lundberg, and R. Tanaka (1976) Integration in descending motor pathways controlling the forelimb in the cat. 2. Convergence on neurones mediating disynaptic corticomotoneuronal excitation. Exp. Brain Res.'26: 521-540.

Kandel, E. R. (1976) Cellular Basis of Behavior, An Introduction to Behavioral Neurobiology, Freeman, San Francisco.

Kandel, E. R., and W. A. Spencer (1968) Cellular neurophysiological approaches in the study of learning. Physiol. Rev. 48: 65-134.

Murakami, F., N. Tsukahara, and Y., Fujito (1977a) Analysis of unitary EPSPs mediated by the newly-formed cortico-rubral synapses after lesion of the nucleus interpositus of the cerebellum. Exp. Brain Res. 30: 233-243.

Murakami, F., N. Tsukahara, and Y. Fujito (1977b) Properties of the synaptic transmission of the newly-formed corticorubral synapses after lesion of the nucleus interpositus of the cerebellum. Exp. Brain Res. 30: 245-258.

Nakamura, Y., N. Mizuno, A. Konishi, and M. Sato (1974) Synaptic reorganization of the red nucleus after chronic deafferentation from cerebellorubral fibers: An electron microscope study in the cat. Brain Res. 82: 298-301.

Smith, A. M. (1970) The effects of rubral lesions and stimulation on conditioned forelimb flexion responses in the cat. Physiol. Behav. 5: 1121-1126.

Thompson, R. F., M. M. Patterson, and T. J. Teyler (1972) 'The neurophysiology of learning. Annu. Rev. Psychol. 23: 73-104.

Thompson, R. F., M. M. Patterson, and W. Berger (1978) Associative learning in the mammalian nervous system. In Brain and Learning. T. J. Teyler, ed., pp. 51-90, Reidel, Dordrecht, Holland.

Toyama, K., N. Tsukahara, K. Kosaka, and K. Matsunami (1970) Synaptic excitation of red nucleus neurons by fibres from interpositus nucleus. Exp. Brain Res. 11: 187-198.

Tsukahara, N. (1978) Synaptic plasticity in the red nucleus. In Neuronal Plasticity, C. W. Cotman, ed., pp. 113-130, Raven Press, New York.

Tsukahara, N. (1981) Synaptic plasticity in the mammalian central nervous system. Annu. Rev. Neurosci. 4, in press.

Tsukahara, N., and Y. Fujito (1976) Physiological evidence of formation of new synapses from cerebrum in the red nucleus following cross-union of forelimb nerves. Brain Res. 106: 184188.

Tsukahara, N., and K. Kosaka (1968) The mode of cerebral excitation of red nucleus neurons. Exp. Brain Res. 5: 102-117.

Tsukahara, N., H. Hultborn, and F. Murakami (1974) Sprouting of cortico-rubral synapses in red nucleus neurons after destruction of the nucleus interpositus of the cerebellum. Experientia 30: 57-58.

Tsukahara, N., H. Hultborn, F. Murakami, and Y. Fujito (1975) Electrophysiological study of formation of new synapses and collateral sprouting in red nucleus neurons after partial denervation. J. Neurophysiol. 38: 1359-1372.

Tsukahara, N., Y. Oda, and T. Notsu (1979) Associative conditioning mediated by the red nucleus in the cat. Proc. Jpn. Acad. Ser. B. 55: 537-541.

Woody, C. D., and G. Brozek (1969) Changes in evoked responses from facial nucleus of cat with conditioning and extinction of an eye blink. J. Neurophysiol. 32: 717-726. 